\title{
Comparação da força dos músculos isquiotibiais antes e após o alongamento e crioterapia
}

\author{
Comparison from the strength of hamstring muscles before and after stretching and cryotherapy \\ Comparación de la fuerza de los músculos isquiotibiales antes y después del estiramiento y la \\ crioterapia
}

Recebido: 14/04/2021 | Revisado: 19/04/2021 | Aceito: 20/04/2021 | Publicado: 04/05/2021

\author{
Vinicius Alexandre Alves da Silva \\ ORCID: https://orcid.org/0000-0001-8938-2882 \\ Universidade Católica de Pernambuco, Brasil \\ E-mail: Alexandrevinicius785@gmail.com \\ Paulo Henrique Altran Veiga \\ ORCID: https://orcid.org/0000-0003-4303-8044 \\ Universidade Católica de Pernambuco, Brasil \\ E-mail: Paulohveiga1@gmail \\ Gabriel Michiles de Assunção Santos \\ ORCID: https://orcid.org/0000-0003-2717-9381 \\ Universidade Católica de Pernambuco, Brasil \\ E-mail: bielmichiles@gmail.com \\ Beatriz Rithiely Henrique Ramos da Silva \\ ORCID: https://orcid.org/0000-0003-3720-4448 \\ Universidade Católica de Pernambuco, Brasil \\ E-mail: beatriz.rithiely@hotmail.com \\ Juliana Bezerra Vieira Costa Cavalcanti \\ ORCID: https://orcid.org/0000-0003-4628-7759 \\ Universidade Católica de Pernambuco, Brasil \\ E-mail: julianabvcc@gmail.com \\ Maria Eduarda Siqueira Veras \\ ORCID: https://orcid.org/0000-0001-9400-2987 \\ Universidade Católica de Pernambuco, Brasil \\ E-mail: dudasveras1@gmail.com \\ Ingrid Holanda Silva \\ ORCID: https://orcid.org/0000-0003-3374-6354 \\ Universidade Católica de Pernambuco, Brasil \\ E-mail: ingridholanda11@outlook.com \\ Patrícia Lopes Ferreira de Lima \\ ORCID: https://orcid.org/0000-0002-5464-5942 \\ Universidade Católica de Pernambuco, Brasil \\ E-mail: patricialopesflima@outlook.com \\ Ellen Raquel Lopes Rodrigues \\ ORCID: https://orcid.org/0000-0002-3375-0401 \\ Universidade Católica de Pernambuco, Brasil \\ E-mail: ellenrlopes5@gmail.com
}

\begin{abstract}
Resumo
O presente estudo tem como objetivo identificar os efeitos imediatos provocados pela técnica de alongamento e da crioterapia na força dos músculos isquiotibiais. Trata-se de um estudo de caráter transversal com ensaio clínico randomizado. Participaram do estudo 26 indivíduos, divididos em dois grupos, o primeiro de alongamento estático $(n=12)$ e segundo de crioterapia $(n=14)$, todos voluntários foram avaliados previamente através do dinamômetro manual isométrico Hand-Held Dynamometry (HHD). Cada voluntário realizou três contrações, obtendo uma média geral dessas contrações, em seguida os voluntários grupo do 1, foram submetidos ao alongamento estático cinco vezes, com a duração de quinze segundos, e o intervalo de trinta segundos entre cada alongamento. O grupo 2 permaneceu com uma bolsa de gelo sobre o ventre médio distal dos músculos isquiotibiais durante 20 minutos e ao final de cada procedimento, de forma imediata passaram por uma reavaliação da força muscular. Nos resultados a população apresentou bastante homogeneidade quanto a avaliação do perfil físico entre os grupos. Quanto a aplicação da técnica de alongamento, encontramos uma redução significativa no desenvolvimento da máxima contração isométrica dos isquiotibiais com um valor de $\mathrm{P}=0,0006$, diferentemente do grupo submetido a crioterapia, o qual demonstrou alteração significativa quando comparado aos valores basais. Com base nos dados obtidos pelo HHD, foi possível concluir que o alongamento de curta duração quando precedido de atividades de força, provoca efeitos
\end{abstract}


adversas no seu desenvolvimento máximo, já a crioterapia não demonstrou nenhuma alteração após sua aplicação. Palavras-chave: Força muscular; Alongamento passivo; Crioterapia.

\begin{abstract}
This scientific article has as its objective to show the immediate effects caused by the stretching technique and cryotherapy in the strength of the hamstring muscles. It is made with a cross sectional study and a randomized clinical trial, with 26 individuals taking part. The individuals were split in two groups, the first one being stretching static $(n=12)$ and the second one cryotherapy $(n=14)$, and all volunteers were previously evaluated using the manual dynamometer isometric Hand-Held Dynamometry (HDD). Each volunteer performed three contractions and had an overall score for those, and then the individuals of both groups were submitted into the techniques destined to their group, and by the end of every procedure, were immediately re-evaluated to check on muscle strength. In the results, the population showed a homogenous response in the evaluation of the physical profile between the groups. In the application of the stretching technique, it was found significant reduction in the development of maximum isometric contraction of the hamstrings with a value of $\mathrm{P}=0,0006$, differently from the group submitted into cryotherapy, which demonstrated significant alteration when compared to the initial value. Based on the data obtained by the HDD, it was possible to conclude that the short term stretching when preceded by activities of strength can cause adverse effects in its maximum development, being that cryotherapy didn't demonstrate any alteration after its application.
\end{abstract}

Keywords: Muscle strength; Passive stretching; Cryotherapy.

\title{
Resumen
}

El presente estudio tiene como objetivo identificar los efectos inmediatos provocados por la técnica de estiramiento y crioterapia sobre la fuerza de los músculos isquiotibiales. Este es un estudio transversal con un ensayo clínico aleatorizado. En el estudio participaron veintiséis individuos, divididos en dos grupos, el primero con estiramiento estático $(n=12)$ y el segundo con crioterapia con $(n=14)$, todos los voluntarios fueron evaluados previamente mediante la dinamometría manual isométrico Hand-Held Dynamometry (HHD). Cada voluntario realizó tres contracciones, obteniendo un promedio general de estas contracciones, luego los voluntarios del grupo 1 y del grupo 2 fueron sometidos a técnicas diseñadas para cada grupo, y al final de cada procedimiento, inmediatamente fueron sometidos a una reevaluación de la fuerza muscular. En los resultados, la población fue bastante homogénea en cuanto a la valoración del perfil físico entre los grupos. En cuanto a la aplicación de la técnica de estiramiento, encontramos una reducción significativa en el desarrollo de la máxima contracción isométrica de los isquiotibiales con un valor de $\mathrm{P}=0,0006$, a diferencia del grupo sometido a crioterapia, que mostró un cambio significativo con respecto a los valores basales. A partir de los datos obtenidos por el HHD, se pudo concluir que el estiramiento de corta duración, cuando es precedido de actividades de fuerza, provoca efectos adversos en su máximo desarrollo, mientras que la crioterapia no mostró cambios tras su aplicación.

Palabras clave: Fuerza muscular; Estiramiento pasivo; Crioterapia.

\section{Introdução}

Todas as fibras musculares possuem unidades contráteis de miofilamentos deslizantes, portanto, uma estrutura bastante hábil quando induzida a um alongamento ou encurtamento. As células do tecido fascial também possuem elementos contráteis, no entanto, essa contração acontece de forma muito tênue, já que este tecido se especializa no revestimento dos músculos para dar forma e separá-los em compartimentos. Essas estruturas citadas acima formam o complexo miofascial, um local em que se desenvolvem normalmente aderências e contraturas, que dificulta o deslizamento entre os tecidos muscular e fascial, afetando o movimento durante toda sua excursão normal, assim como o desenvolvimento da força. (Silva, 2018).

A capacidade de o corpo mover-se livremente para a execução das atividades cotidianas ou recreativas, torna-se necessário, para a manutenção da saúde tecidual do corpo. (Kisner \& Colb, 2016). Nessa perspectiva, é fundamental a integridade dos tecidos que podem restringir essa mobilidade, que são: músculo, fáscia e a pele. Assim, quando essas estruturas são submetidas a um alongamento, ocorre alteração elástica ou plástica. As técnicas de alongamento, para ser consideradas como efetiva, com um ganho de elasticidade ou plasticidade dos tecidos, acontecem numa faixa de quinze a trinta segundos, acima disso os pesquisadores identificaram que não ocorre nenhum ganho a mais. (Bandy; Irion \& Briggler, 1997; Milazzotto et al, 2009).

Com o aumento do interesse da população em realizar atividades esportivas, esses indivíduos se sentiram na necessidade de cada vez mais incorporar gestos esportivos, que exigem uma maior mobilidade, ou seja, uma integridade do 
complexo miofascial. Sendo assim, se mostrou a necessidade de participantes amadores ou recreativos em adotar programas de exercícios que antecedem o gestual esportivo, entre eles o alongamento, onde muitos executam com a crença de que vai aumentar a flexibilidade, reduzir o risco de lesões e melhora no desempenho esportivo. (Fjertad et al., 2018)

Da mesma forma, a crioterapia também é muito utilizada na atualidade para atenuar ou para a prevenção de lesões. Sabe-se que a crioterapia tem efeitos fisiológicos de vasoconstrição, redução da temperatura da pele e do tecido subcutâneo, diminui a excitabilidade das fibras nervosas periféricas e consequentemente altera o limiar de dor, reduz a taxa metabólica, o que diminui a resposta inflamatória. (Karvat et al., 2016). Porém, apesar da crioterapia ser um ótimo e já conhecido elemento físico bem fundamentado na literatura em relação a sua eficácia na prevenção de lesões, por causa da hipóxia secundária, ainda não existe consenso entre os seus efeitos em relação a força dos músculos quando tratado com essa técnica. (Klein, 2015).

O equilíbrio entre os grupos musculares é fundamental para que ocorra um movimento harmônico, suave, coordenado e que minimize a probabilidade de lesões musculares devido ao seu desequilíbrio. Desequilíbrio esse que promove diversas alterações no membro inferior, em particular no joelho. Essas modificações são resultantes da desarmonia na relação I/Q, ou seja, entre os flexores e extensores do joelho que pode ser decorrente de lesões, ações ou técnicas que provoquem alteração muscular previamente a realização da atividade (Mortari; Manica \& Pimentel, 2009).

Dessa forma, tanto a crioterapia quanto o alongamento são técnicas amplamente utilizadas pelos terapeutas. Contudo, a compreensão dos efeitos destes procedimentos sobre a força muscular, ainda são bastante intrincados entre os profissionais que fazem sua utilização. Além disso, a região dos isquiotibiais foi escolhido de forma intencional, por ser uma região central de discussões devido à necessidade de debruçar mais sobre o seu comportamento frente a situações de exposição, e tendo em vista que se trata de um local com elevado índice de lesões, associadas a um longo período de recuperação e pela possibilidade de desenvolver patologias crônica na região do joelho. Por esses motivos, este estudo busca quantificar e identificar o comportamento da força muscular dos isquiotibiais imediatamente após a aplicação da crioterapia e realização dos alongamentos, a fim de entender melhor o seu comportamento e instigar mais estudos sobre a temática.

\section{Metodologia}

O estudo é de caráter transversal com ensaio clínico randomizado. A partir do cálculo amostral o objetivo inicial era compor a pesquisa com 30 indivíduos, contudo, devido a interrupção das atividades universitária por conta da pandemia SarsCov-2, a coleta se restringiu aos meses de fevereiro a março de 2020. Dessa forma, a população do estudo se limitou a 24 participantes.

Os participantes do estudo foram constituídos, por indivíduos com idade entre 18 a 28 anos, independentemente do sexo. Foram utilizados como critérios de inclusão: indivíduos hígidos, cognitivamente competentes e que aceitassem assinar o Termo de Consentimento Livre e Esclarecido (TCLE). Como critérios de exclusão: foram considerados os indivíduos que apresentaram história de lesão do Isquiotibiais, dor no joelho nos últimos seis meses ou que não tivesse coordenação muscular suficiente para realizar o teste de força.

Após assinatura do TCLE, foi aplicado um questionário presente no aplicativo My Sp Tech (Beta), aplicativo esse necessário para a visualização gráfica da força muscular em tempo real, contendo perguntas como, idade, peso e altura. Em seguida, todos os participantes foram submetidos à avaliação da força dos músculos isquiotibiais, através do dinamômetro manual isométrico Hand-Held Dynamometry (HHD), instrumento esse que possui alta confiabilidade, além de ser um equipamento de fácil manuseio e de baixo custo quando comparado a dinamômetro isocinético, para avaliação de torque muscular (Almeida et al, 2019).

Para a avaliação da força dos isquiotibiais, foi detalhado aos voluntários como seriam realizados a coleta, esse período já serviu como critério de repouso de cinco minutos para padronização antes de captar a primeira contração. O posicionamento 
de todos indivíduos foi em decúbito ventral, com os membros inferiores em cadeia cinemática aberta, o quadril em posição neutra e $45^{\circ}$ de flexão do joelho. Após o posicionamento, foi fixado duas cintas, uma no quadril para estabilizar e outra no tornozelo, a fim que limite o movimento de flexão do joelho até $45^{\circ}$, e o terapeuta solicitava ao voluntário que realizasse uma flexão do joelho em seu lado dominante. Nesse momento, o terapeuta posiciona o dinamômetro sobre o calcanhar, de forma que o voluntário aplicasse sua força máxima contra o equipamento durante sete segundos para, assim, realizar a avaliação.

Foram captadas três contrações, utilizando a média geral destas contrações como dados para o estudo. Neste momento, os indivíduos foram divididos aleatoriamente em dois grupos pelo sorteio na moeda: o grupo um foi composto por 12 indivíduos submetidos ao alongamento e mais 14 indivíduos no grupo dois, submetidos a crioterapia. As forças dos isquiotibiais de todos os indivíduos foram avaliadas novamente depois de um minuto e meio de descanso, após o alongamento e do mesmo jeito no grupo da crioterapia.

A técnica de alongamento foi realizada com o voluntário em decúbito dorsal, sendo que o terapeuta utiliza a mão cranial para impedir uma flexão do joelho, enquanto a mão caudal leva todo o membro inferior para uma flexão do quadril. Dessa forma, alonga os isquiotibiais até seu comprimento máximo, e assim, identifica o grau de flexibilidade desta musculatura. (Kisner \& Colb, 2016). O alongamento estático foi realizado cinco vezes, com a duração de quinze segundos, e com o intervalo de trinta segundos entre cada alongamento. (Arjang et al, 2019). A crioterapia foi realizada com o voluntário em decúbito dorsal, e o terapeuta posicionou uma bolsa de gelo sobre o ventre médio distal dos músculos isquiotibiais, mantendo nesta posição durante 20 minutos. (Klein, 2015). Ambas as técnicas, foram realizadas em um único atendimento, a fim de identificar os efeitos imediatos, ou seja, assim que a técnica foi finalizada o HHD já era posicionado para mensuração.

Para uniformizar as medidas, todas as avaliações foram feitas pelo pesquisador principal do estudo, sendo capacitado para uma captação apropriado pelo seu orientador, o qual tem reconhecimento nacional nas abordagens musculoesqueléticas. Além disso, foi utilizado uma bolsa plástica na medida especifica a qual não necessitou de adaptações, devido a homogeneidade da altura dos participantes.

As variáveis contínuas foram apresentadas como média e desvio padrão (técnicas de estatística descritivas). Para testar a suposição de normalidade das variáveis envolvidas no estudo, foi aplicado o teste Komogorov-Smirnov. Foi verificada parametricidade nas avaliações das técnicas de alongamento e crioterapia. Após a confirmação da parametricidade, para a discriminação das diferenças (comparação das médias dos resultados), entre os grupos (alongamento e crioterapia), foi utilizado o teste $t$ de student pareado. Para se avaliar a diferença entre resultados antes e depois dos tratamentos nos grupos, foi utilizado o teste Anova One Way. Considerou-se o nível de significância $p \leq 0,05$. Os dados foram digitados na planilha Excel 2015® e o software utilizado para a obtenção dos cálculos estatísticos foi o GraphPad Prism 4 ®.

Indo de acordo com as recomendações éticas que guiam pesquisas com seres humanos, o presente estudo seguiu o código de ética de fisioterapia, somado a resolução 466/12 da comissão nacional de ética em pesquisa, recebendo parecer favorável n ${ }^{\circ}$ 140918/2018. A pesquisa ofertou riscos mínimos aos voluntários, pois o sigilo foi assegurado pelos autores, já que todo processo foi individualizado e com preservação de sua identidade.

\section{Resultados}

A amostra deste estudo foi composta por 26 indivíduos de ambos os sexos, havendo a prevalência do sexo feminino, que correspondeu a $80 \%$ da população estudada.

Na Tabela 1 estão expostas a caracterização do perfil dos voluntários, no que se diz respeito à idade, peso e altura, alcançando respectivamente uma média de 21,46 e com desvio padrão de $\pm 1,44$ sob o valor total; com faixa etária entre 20-25 anos, com relação ao peso e altura os dois grupos também tiveram resultados bem semelhantes, mesmo não participando de 
grupos específicos. Sendo assim, os valores de cada tópico avaliado ultrapassaram $p$ valor $\leq 0,05 \%$, demonstrando o quão homogêneo foram os indivíduos que compõem cada grupo.

Tabela 1. Caracterização do perfil fisiológico dos participantes do estudo ( $\mathrm{n}=26)$.

\begin{tabular}{l|l|l|l}
\hline & $\begin{array}{l}\text { IDADE } \\
\text { (Media/DP) }\end{array}$ & $\begin{array}{l}\text { PESO } \\
\text { (Media/DP) }\end{array}$ & $\begin{array}{l}\text { ALTURA } \\
\text { (Media/DP) }\end{array}$ \\
Alongamento & $21,46 / 1,44$ & $61,19 / 13,39$ & $1,65 / 0,09$ \\
Crioterapia & $21,50 / 1,50$ & $60,75 / 13,77$ & $1,65 / 0,10$ \\
$\begin{array}{l}\text { P valor } \\
\text { (Alongamento x. Crio) }\end{array}$ & $21,43 / 1,45$ & $61,57 / 13,56$ & $1,64 / 0,09$ \\
\hline
\end{tabular}

Legenda: DP = desvio padrão; Diferença estatisticamente significante p-valor $\leq 0,05 \%$. Fonte: Autores (2020).

Na Tabela 2, é possível identificar que os dois grupos apresentaram uma leve diminuição da força muscular dos flexores do joelho após as intervenções, quando comparado aos valores basais. No entanto, em normas estatísticas é possível comprovar, devido ao P-valor, que apenas o grupo submetido ao alongamento demonstrou diferença estatisticamente significante, antes e após a intervenção, obtendo-se p 0,0006. Quando observado as variações entre as respostas e a porcentagem de confiança entre os resultados pelo R Squared, o grupo submetido ao alongamento teve um R de 76,34\%, e o grupo de crioterapia com um R de 22,99\% de variação, o que totalizou em um R de variação na diferença de resposta entre os dois grupos de 90,97\%, resultado esse que demonstra uma alta confiança. Dessa forma, fica claro que após a aplicação de crioterapia não há alteração no pico do torque dos isquiotibiais, diferentemente da técnica de alongamento, que promove um decréscimo do mesmo.

Tabela 2. Comparação dos resultados Pré e Pós realização das técnicas de Alongamento e Crioterapia, entre os indivíduos do estudo.

\begin{tabular}{ccccc}
\hline TECNICA DE & PRÉ & PÓS & Teste t \\
TRATAMENTO & Média \pm DP & Média \pm DP & $\begin{array}{c}\text { R } \\
\text { Valor de p }\end{array}$ & Squared \\
\hline Alongamento & $24,34 \pm 5,48$ & $23,28 \pm 5,58$ & $0,0006^{*}$ & 0,7634 \\
Crioterapia & $22,81 \pm 5,61$ & $21,67 \pm 6,32$ & 0,0705 & 0,2299 \\
$\begin{array}{c}\text { Alongamento } \mathbf{x} \\
\text { Crioterapia }\end{array}$ & --- & --- & $0,0001^{\Psi}$ & 0,9097 \\
\hline
\end{tabular}

Legenda: DP= desvio padrão; Teste de normalidade de Komogorov Smirnov; Teste t* para amostras pareadas; One-Way Anova ${ }^{\Psi ;}$ Diferença estatisticamente significante p-valor $\leq 0,05 \% *$. Fonte: Autores (2020).

\section{Discussão}

Apesar de estar bem estabelecido na literatura que o alongamento é uma técnica que não proporciona o aumento da força, encontra-se uma grande divergência no que se diz a respeito se ela provoca o decréscimo ou simplesmente não altera a força muscular. Quanto a crioterapia, é um procedimento amplamente utilizado como meio de causar analgesia e que acompanha todo processo de recuperação, contudo há poucos estudos correlacionando com a força musculoesquelética. No 
entanto, os achados desse estudo; nos afirmam que o alongamento estático com os critérios estabelecido nesse estudo provocou um efeito agudo no desempenho máximo da contração isométrica dos isquiotibiais, diferentemente da crioterapia.

Vários estudos vêm mostrando os achados de como o alongamento estático provoca repercussões negativas sobre os sistemas central e periférico para desenvolver a força muscular máxima. (Chaabene et al, 2019). No entanto, essas repercussões quando falamos de alongamentos de curta duração, ou seja, abaixo de 45 segundos, se tornam insignificantes. Como demonstrado no estudo de Palmer et al, 2019, em que não houve alteração na taxa de ativação muscular após a técnica, assim como nos achados de Matsuo et al, 2013, o qual não encontrou alteração significativa na rigidez do sistema musculotendíneo. Dessa forma, não se encontra na literatura uma justificativa plausível para explicar os mecanismos subjacentes responsáveis pelas deficiências induzidas do alongamento estático breve nas atividades subsequentes de força.

Os resultados obtidos neste estudo, nos mostraram que o grupo submetido ao alongamento estático de 15 segundos sofreu um declínio no desempenho da força flexora do joelho, com uma redução estatisticamente significativa, como demonstrado no valor de P 0,0006, o que corroborou com os achados de Franco et al, 2008; Winchester et al, 2009 \& Gomes et al, 2010 no qual também obteve redução significativa. No entanto, discordou com os achados de Reid et al, 2018; Blazevich et al., 2018, que não encontrou alterações relevantes em alongamento de curta duração e afirma uma direta relação entre a dose e o efeito.

Não são tão claros, até o momento, as repercussões terapêuticas da crioterapia em relação à força muscular. No entanto, algum estudo mais recente vem demonstrando uma conclusão bem semelhante quando a inalteração da força muscular, como nos estudos de Mortari et al, 2009; Dohnert et al, 2017, que afirmam que os métodos usuais de crioterapia, como no presente estudo, não alteram o desempenho motor, pois não modifica a percepção da carga imposta. O que foi de acordo com as observações dos dados desse grupo no presente estudo, em que não foi verificado modificações estatisticamente significativas. No entanto, como não existe uma padronização de concordância entre os resultados da crioterapia na força, alguns estudos discordaram, como nos estudos de Sellwood et al, 2007; Torres et al, 2015, em que observaram declínio do desempenho muscular e concluíram que os mecanismos vão desde redução da velocidade da condução nervosa até lentidão dos processos químicos no interior do músculo.

\section{Conclusão}

Diante da situação estudada, foi possível concluir que a técnica de alongamento estático dos isquiotibiais, dentre os padrões proposto pelo estudo, foi capaz de gerar repercussões negativas do desempenho máximo da contração isométrica, e que a aplicação de crioterapia não provoca alterações importantes nos valores pós avaliação.

Contudo, por se tratar de um recurso de fácil aplicação, baixo custo e de grande utilização na prática clínica diária, se faz necessário realizar mais estudos, questionando outros fatores associados, tendo em vista que, o presente estudo se deteve a avaliar apenas o efeito imediato das técnicas, fatores esses que se diz respeito ao tempo em que permanece a redução da força após o alongamento estático, efeito tardio e acumulativo desses dois recursos ao longo de um protocolo terapêutico, ao invés de simplesmente banir sua utilização.

O estudo apresentou alguns pontos negativos, quanto a amostra do estudo ser bastante reduzida, não haver distinção de sexo e nem condições especifica para sua classificação quanto a ser sedentário ou praticante de atividade física, no entanto, devido à escassez de estudos voltados para essa temática, a presente pesquisa serve como fomento de base teórica e metodologia a ser melhor adaptada em pesquisas futuras, a fim de, preencher as lacunas apresentadas no estudo e proporcionar melhor compreensão dos efeitos das técnicas abordadas sobre o tecido muscular. 


\section{Referências}

Almeida, G. P. L., Alban, T. R., \& Melo, A. K. P. (2019). Dinamômetro portátil identifica assimetrias no torque do músculo quadríceps após reconstrução do ligamento cruzado anterior. Cirurgia do joelho, traumatologia esportiva, artroscopia: jornal oficial da ESSKA, 27 (8), 2494-2501. 10.1007/0167-018-5245-3

Arjang, N., Mohsenifar, H., Amiri, A., Dadgoo, M., \& Rasaeifar, G. (2019). Os efeitos imediatos do alongamento de facilitação neuromuscular estática versus proprioceptiva com cinesiologia com flexibilidade de isquiotibiais em jogadores adolescentes de taekwondo. Journal of Clinical Physiotherapy Research, 3(4), 132-138. https://doi.org/10.22037/jcpr.v3i4.23941

Bandy, D. W., Irion, J. M., \& Briggler, M. (1997). O efeito do tempo e da frequência do alongamento estático na flexibilidade dos músculos isquiotibiais. Fisioterapia, 77(10), 1090-1096. 10.1093/ptj/77.10.1090.

Blazevich, A. J., Gill, N. D., Kvorning, T., Kay, A. D., Goh, A. G., Hilton, B., Drinkwater, E. J., \& Behm, D. G. (2018). No effect of muscle stretching within a full, dynamic warm-up on athletic performance. Med. Sci. Sports Exercises, 50(6), 1258-1266. https://doi.org/10.1249/MSS.0000000000001539

Chaabene, H., Behm, D. G., Negra, Yassine \& Granacher, U. (2019). Acute effects of static stretching on muscle strength and power: an attempt to clarify previous caveats. Rev. Frontiers in Physiology. 10. https://doi.org/10.3389/fphys.2019.01468.

Dohnert, M. B., Oliveira, M. S., \& Hoffmannc, R. F. (2017). Efeito agudo da crioterapia e diatermia na flexibilidade e força muscular de isquiotibiais. Rev. Ciência e Saúde, 10(2), 89-95. https://doi.org/10.15448/1983-652X.2017.2.24474

Fjerstad, B. M., Hammer, R. L., Hammer, A. M., Connolly, G., Lomond, K. V., \& O'connor, P. (2018). Comparação de dois procedimentos de alongamento estático na flexibilidade e força adutora do quadril. Revista internacional de ciência do exercício, 11 (6), 1074 - 1085. https://www.ncbi.nlm.nih.gov/pmc/articles/PMC6179425/.

Franco, B. L., Signorelli, G. R., Trajano, G. S., \& Oliveira, C. G. (2008). Acute effects of different stretching exercises on muscular endurance. Journal of Strength Conditioning Research, Connecticut, 22(6), 1832-1837. 10.1519 / JSC.0b013e31818218e1.

Gama, H. S., Yamanishi, J. N., Gallo, L. H., Valderramas, S. R., \& Gomes A. R. S. (2018). Exercícios de alongamento: uma atualização da prescrição e dos efeitos na função musculoesquelética, 26 (1). https://hdl.handle.net/1884/42148.

Gomes, T. M., Simão, R., Marques, M. C., Costa, P. B., \& da Silva Novaes, J. (2010). Acute Effects of Two Different Stretching Methods on Local Muscular Endurance Performance. Journal of Strength Conditioning Research, Connecticut, 25(3), 745- 752. 10.1519/JSC.0b013e3181cc236a.

Karvat, J., Kakihata, C. M. M., Vieira, L., Antunes, J. S., Ribeiro, L. F. C., \& Bertolini, G.R.F. (2016). Avaliação da nocicepção e edema em modelo experimental de compressão do nervo isquiático em ratos Wistar tratados com crioterapia. Revista Dor. 17(3), 210-4. https://doi.org/10.5935/18060013.20160073

Kisner, C., \& Colby, L. (2016). A. Exercícios terapêuticos: fundamentos e técnicas (6a ed.). Manole.

Klein, C. C. (2015). A crioterapia e o calor superficial no ganho de flexibilidade nos músculos isquiotibiais. Trabalho de Conclusão de Curso. Universidade do Vale do Taquari. Centro Univates, Lajeado, RS. http://hdl.handle.net/10737/2055.

Matsuo, S., Suzuki, S., Iwata, M., Banno, Y., Asai, Y., Tsuchida, W \& Inoue, T. (2013). Acute effects of different stretching durations on passive torque, mobility, and isometric muscle force. J. Strength Cond. Res, 27, 3367-3376. 10.1519/JSC.0b013e318290c26f.

Milazzotto, M. V., Corazzina, L. G., \& Liebano, R. E (2009). Influência do número de séries e tempo de alongamento estático sobre a flexibilidade dos músculos isquiotibiais em mulheres sedentárias. Rev. Brasileira de Medicina do Esporte, 15(6), 420-423. https://doi.org/10.1590/S1517-86922009000700003.

Mortari, D. M., Mânica, A. P., \& Pimentel, G. L (2009). Efeitos da crioterapia e facilitação neuromuscular proprioceptiva sobre a força muscular nas musculaturas flexora e extensora de joelho. Rev. Fisioterapia e Pesquisa, 16(4), 329-334. https://doi.org/10.1590/S1809-29502009000400008.

Palmer, T. B., Pineda, J, G., Cruz, M. R \& Agu-Udemba, C. C. (2019). Duration-dependent effects of passive static stretching on musculotendinous stiffness and maximal and rapid torque and surface electromyography characteristics of the hamstrings.J. Strength Cond. Res, 33, 717-726. 10.1519/JSC.0000000000003031.

Reid, J. C., Greene, R., Young, J. D., Hodgson, D. D., Blazevich, A. J., \& Behm, D. G. (2018). The effects of different durations of static stretching within a comprehensive warm-up on voluntary and evoked contractile properties. Eur. J. Appl. Physiol. 118, 1427-1445. https://doi.org/10.1007/s00421-018-3874-3.

Torres, R., Silva, F., Pedrosa, V., Ferreira, J., \& Lopes, A. (2015). The acute effect of cryotheray on muscle strength and shoulder proprioception. J. Sport Rehabil. 26(6), 497-506. https://doi.org/10.1123/jsr.2015-0215 